\title{
Transperineal Parallel Biopsy of the Prostate: A New Approach of Tissue Sampling for Precision Medicine
}

Min Qu, ${ }^{1, *}$ Bijun Lian, (D) ${ }^{1,2, *}$ Yan Wang, ',* Wenhui Zhang,' Feng Zhu, ',3 Tao Wang, ${ }^{4}$ Xiaodong Yue, ${ }^{5}$ Zepeng Jia,' Huan Chen,' Husheng Li,'

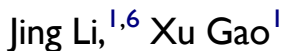

'Department of Urology, Changhai Hospital, Second Military Medical University, Shanghai, People's Republic of China; ${ }^{2}$ Department of Urology, The 903th PLA Hospital, Hangzhou, People's Republic of China; ${ }^{3}$ Department of Urology, Tianyou Hospital, Tongji University, Shanghai, People's Republic of China; ${ }^{4}$ Department of Urology, First Affiliated Hospital of Zhengzhou University, Zhengzhou, People's Republic of China; ${ }^{5}$ Shanghai Institute for Advanced Communication and Data Science, Shanghai University, Shanghai, People's Republic of China; ${ }^{6}$ Center for Translational Medicine, Second Military Medical University, Shanghai, People's Republic of China

*These authors contributed equally to this work

Correspondence: Jing Li

Center for Translational Medicine, Second Military Medical University, No. 800

Xiangyin Road, Yangpu District, Shanghai, 200438, People's Republic of China

Tel +86 02I-3II6I7I8

Email ljing@smmu.edu.cn

Xu Gao

Department of Urology, Changhai

Hospital, No. 168 Changhai Road, Yangpu

District, Shanghai, 200438, People's

Republic of China

Tel +86 02I-3II6I7I7

Email gaoxu.changhai@foxmail.com
Purpose: Through an observational study to present a new approach for obtaining highquality samples for the targeted therapy of prostate cancer.

Patients and Methods: Parallel biopsy, which was defined as collecting the tissue from the same site by two biopsies, was performed on patients with elevated PSA. Each tissue was stained by ink to identify the pathological characteristics, including Gleason score and tumor tissue ratio. Kendall tau-b test and intraclass correlation coefficient test were used to compare the consistency between each paired sample. Then, based on the pathology of the biopsies, high-quality tissues would be selected for sequencing, and PyClone model was used to track the clonal evolution.

Results: In total, 252 pairs of biopsies were collected. The consistency of Gleason score between each paired biopsy is $0.777(p<0.01)$, and the consistency of tumor tissue ratio is $0.853(p<0.01)$. With the application of parallel biopsy, on average five nonsynonymous mutations could be identified in patients with castration-resistant prostate cancer. Six out of eight had at least one biology-relevant alteration in patients, guiding further treatment. Meanwhile, clonal evolution was constructed to investigate the progress of tumor.

Conclusion: Parallel biopsy is a reliable approach to collect high-quality tissue and shows potential application in precision medicine.

Keywords: prostate cancer, prostate biopsy, next-generation sequencing, clonal evolutionary tree, precision medicine

\section{Introduction}

Prostate cancer (PCa) is the most frequently diagnosed solid malignancy and the third leading cause of cancer related-death in males in the United States. ${ }^{1}$ Due to the multifocal nature of the disease, it is regarded as one of the most heterogeneous tumors. ${ }^{2}$ Although plenty of researches have been published, investigating the genetic sequencing results of a large series of $\mathrm{PCa}$, the common limitation is that each sample is from one focus per patient, lacking the characteristic of heterogeneity of the cancer. ${ }^{3-5}$ On the other hand, there is still controversy regarding how PCa progresses and becomes resistant to castration in late stage, which stimulates the exploration of the mechanism focusing on the tumor evolution and further precision treatment. ${ }^{6}$ Now, a key to understand the heterogeneity is to reveal the complicated genomic diversity and variability within a tumor by next-generation sequencing (NGS) technologies of tumor tissue. Meanwhile, circulating tumor cells (CTCs) and urine exosomes also show great potential for future research. ${ }^{7,8}$ 
Generally, there are three ways to acquire samples: tissue from radical prostatectomy, transurethral prostate resection and prostate biopsy. The critical feature of sampling of $\mathrm{PCa}$ is to maintain a certain temporality and spatiality. Radical prostatectomy can collect precise multifocal tissue, ${ }^{9}$ but there is a lack of temporality. Tissue from transurethral prostate resection is short of spatiality, beside being troubled by the validity of sequencing results. Prostate biopsy is considered as the best approach, including both temporality and spatiality of tissue sampling, and is a main method for cancer detection, monitoring and management, which is commonly performed in clinics, in Europe and the United States. ${ }^{10}$ Fresh frozen $(\mathrm{FF})$ samples from which can be extracted more DNA are considered to give better NGS results than formalin-fixed paraffin-embedded (FFPE) samples. ${ }^{11,12}$

In order to improve the sequencing results by providing pathological reference, we firstly present parallel biopsy of the prostate which refers to collecting the tissue from the same site of the prostate by two biopsies. We propose that these two tissues from the same site share the same pathological and biological information. One of the samples was sent for pathological analysis, while the other one was frozen immediately. With reference to pathological result, a sample with high quality will be selected for sequencing. We hope that parallel biopsy will support the construction of the tumor evolutionary tree and show potential application in precision medicine in the future.

\section{Patients and Methods}

\section{Patients}

To ensure that the puncture biopsies contained tumor tissues for comparison, high-risk prostate cancer patients (PSA of higher than $20 \mathrm{ng} / \mathrm{mL}$ ), who may have higher tumor burden, were preferred for this study. All the patients met the indication of prostate biopsy in guidelines ${ }^{13}$ and had not previously had a prostate biopsy. Informed consent for this observational study was obtained, and research biopsy was performed under a study protocol approved by the Changhai Research Ethics Committee.

\section{Biopsy Procedure}

The BK Pro Focus 2202 and BARD Max-CORE Disposable Core Biopsy Instrument were used for the conventional process. The patients were prepared in the lithotomy position. The perineal area was well disinfected and draped before performing biplane transrectal ultrasonography. Firstly, we measured the diameter of the prostate. And then
12 sites ( 6 in peripheral zone, 4 in transitional zone and 2 in central zone) were chosen for biopsy. Two parallel biopsies were performed in each site of the prostate and were sent for pathological diagnosis. All the biopsies were performed by an experienced urologist, and pathological results were analyzed by an experienced pathologist. All the puncture biopsies were performed under local anesthesia.

\section{Statistical Analysis}

SPSS version 22 (SPSS Pty. Ltd, USA) software was used to compare the consistency of the two biopsies. Kendall tau-b test was used for ordered categorical data. Intraclass correlation coefficient test was used for measurement data. The test level was set $\alpha=0.05$, and a $P<0.05$ was considered statistically significant.

\section{Clinical Application and Verification Sample Processing and DNA Extraction}

Biopsy DNA was extracted using AllPrep MinElute Kit (Qiagen) from selected corresponding biopsies. DNA concentration was quantified using a Qubit fluorometer and the Qubit dsDNA HS (High Sensitivity) Assay Kit (Invitrogen, Carlsbad, CA, USA). The size distribution of genomic DNA was assessed using an Agilent 2100 BioAnalyzer and the DNA HS kit (Agilent Technologies, Santa Clara, CA, USA).

\section{Target Panels Design and Sequencing}

The construction of targeted panels was designed with Ampliseq Designer software and covered the coding regions of 612 genes or 240 genes (named Pro240-Panel) frequently mutated in prostate cancer and other common solid tumors. These panels used GRCh37 as references and were sequenced on an Illumina Next 500 platform (Illumina Inc, San Diego, CA, USA).

\section{Whole-Exome Sequencing (WES)}

Whole-exome capture libraries were constructed from 100 ng per sample as input material for the DNA library preparations. Following the Truseq Nano DNA HT Sample Prep Kit (Illumina, USA) protocol, genomic DNA was sheared to a median size of $350 \mathrm{bp}$, then end repair, phosphorylation and ligation to barcoded sequencing adaptors. According to the manufacturer's instructions, genomic libraries were quantified using quantitative PCR and pooled for enrichment by hybridization either using the TruSeq Exome Enrichment Kit (Illumina) or a custom SeqCap EZ Library (Roche NimbleGen, Madison, WI, USA). 


\section{Targeted Gene Sequencing}

The prostate gene panel included 612 genes chosen because of the mutation frequency discovered in large prostate cancer cohorts. The list of panel genes is available in Supplementary Table 1. The libraries were constructed using the KAPA HTP Library Preparation Kit. The regions of interest were captured by the customized probe (SeqCap EZ Choice, Roche Nimblegen, Inc., Madison, WI, USA). Paired-end reads were generated by Nextseq 500 Sequencer.

\section{Bioinformatics Analysis}

The fastq files were quality-checked by fastqc and then mapped to the GRCh37 human reference genome by BWA-MEM aligner. Individual BAM files contain reads aligned to the human genome with quality scores recalibrated using Genome Analysis Toolkit (GATK)'s Table Recalibration tool. All sites potentially harboring small insertions or deletions in either the tumor or the matched normal were realigned using GATK. We used mutect and varscan 2 to call somatic mutations in biopsies. The vcf files were annotated by annovar before subsequent advanced analysis. FACETS ${ }^{14}$ were used to detect copy number variation and tumor purity.

\section{Clone Evolution Analysis}

We used PyClone, ${ }^{15}$ a model to infer the clonality by the Bayesian clustering method, to track the clonal evolution of cancers. The somatic mutations in the exon region with depth higher than $80 \mathrm{X}$ were input to PyClone. Each mutation input contains the major and minor copy number variation from FACETS. We used the PyClone beta binomial model with the "parental_copy_number" option to run the PyClone algorithm. Clonevol ${ }^{16}$ was used to visualize the clonal evolution by phylogenetic tree.

\section{Results \\ Clinical Characters}

In total, 23 patients with elevated PSA had a transperineal parallel biopsy of the prostate. The clinical characteristics are shown in Table 1. Nineteen patients had 12 parallel biopsies as planned before, and the other four had 6 parallel biopsies due to pain intolerance during the procedure. None of them had any adverse events after prostate biopsy. A total of 252 pairs parallel biopsies were analyzed.

\section{Pathological Review}

Pathological reports were presented with Gleason score and tumor tissue ratio for each biopsy; 20 of the 23 patients were diagnosed as $\mathrm{PCa}$, and the other 3 were benign prostatic hyperplasia (BPH). The pathological results are shown in Figure 1. The consistency (Kendall consistency coefficient) of Gleason score between the two parallel biopsies was $0.777(p<0.01)$, and the consistency (intraclass correlation coefficient) of tumor tissue ratio between the two biopsies was $0.853(p<0.01)$.

\section{Application in Precision Medicine}

Based on the high consistency of two parallel biopsies, we proposed to select samples with high quality for clinical NGS (Figure 2). In our preliminary result, eight samples from patients with castration-resistant prostate cancer (CRPC) were sequenced by using our customized gene panel to facilitate the clinical treatment. The basic data statistics and sequencing results are provided in Supplementary Table 2.

We identified about five nonsynonymous mutations (ranging from 1 to 9) in each CRPC sample. Six out of eight had at least one biology-relevant alteration which could guide clinical treatment. Interestingly, we detected AR mutants in two patients, and each patient had two significant hotspot mutations of AR (W742X, T878A), demonstrating the selective pressure of castration in patients with CRPC. Besides, we discovered other driver genes in other patients, such as CDK12, MLH1 and Jak1, which provided guidance for further clinical decisions (Figure 3).

On the other hand, parallel biopsy was applied to decipher the genetic mechanism of intratumor heterogeneity. We performed whole-exome sequencing form the different site of primary tumor by parallel biopsy in one specific patient. Based on deep sequencing, we selected 342 somatic variants including 125 nonsynonymous somatic point mutations (Supplementary Table 3) into the PyClone, and a phylogenetic tree of the tumor regions was constructed by clonevol. All mutations were clustered, and the clones were ordered. Bell plot can present the dynamics of clonal subpopulations in the sample over time, and sphere of cells can present the subpopulation of cells and the proportion in the sample by plotting a total of one hundred cells. The annotated node-based and branch-based trees describe the clonal relationships and seeding patterns between cancers in three regions. The branch length correlates with the number of mutations of the clones, and the marked gene is a driver event in the evolution. In this patient, mutations in PTEN was an early clonal event. Conversely, KMT2C in DHL3, DHL4 and 
Table I Clinical Characters of Patients

\begin{tabular}{|c|c|c|}
\hline & \multicolumn{2}{|c|}{ Patients } \\
\hline & Pathological Biopsy & Parallel Biopsy \\
\hline Total number & \multicolumn{2}{|c|}{23} \\
\hline Age $(y)$ & \multicolumn{2}{|c|}{68 (IQR: 64-72) } \\
\hline PSA (ng/mL) & \multicolumn{2}{|c|}{62.6 (IQR: 33.9-I57.0) } \\
\hline \multicolumn{3}{|l|}{ Clinical T $(n, \%)$} \\
\hline$\leq T_{2 c}$ & \multicolumn{2}{|c|}{$8(34.8)$} \\
\hline$T_{3 a}$ & \multicolumn{2}{|c|}{$4(17.4)$} \\
\hline $\mathrm{T}_{3 \mathrm{~b}}$ & \multicolumn{2}{|c|}{$10(43.5)$} \\
\hline $\mathrm{T}_{4}$ & \multicolumn{2}{|c|}{ I (4.3) } \\
\hline \multicolumn{3}{|l|}{ Clinical N } \\
\hline $\mathrm{N}_{0}$ & \multicolumn{2}{|c|}{$14(60.9)$} \\
\hline$N_{1}$ & \multicolumn{2}{|c|}{$9(39.1)$} \\
\hline \multicolumn{3}{|l|}{ Clinical M } \\
\hline$M_{0}$ & \multicolumn{2}{|c|}{$16(69.6)$} \\
\hline$M_{1}$ & \multicolumn{2}{|c|}{$7(30.4)$} \\
\hline \multicolumn{3}{|l|}{ Biopsy mode (n, \%) } \\
\hline $6+6$ & \multicolumn{2}{|c|}{$4(17.4)$} \\
\hline $12+12$ & \multicolumn{2}{|c|}{$19(82.6)$} \\
\hline Total biopsy cores & \multicolumn{2}{|c|}{252} \\
\hline \multicolumn{3}{|l|}{ Pathological result (n, \%) } \\
\hline $\mathrm{PCa}$ & \multicolumn{2}{|c|}{$20(87.0)$} \\
\hline $\mathrm{BPH}$ & \multicolumn{2}{|c|}{$3(13.0)$} \\
\hline \multicolumn{3}{|l|}{ Single biopsy pathology ( $n, \%)$} \\
\hline $\mathrm{PCa}$ & $172(68.3)$ & $169(67.1)$ \\
\hline $\mathrm{BPH}$ & $80(31.7)$ & $83(32.9)$ \\
\hline \multicolumn{3}{|l|}{ Gleason Grade (n, \%) } \\
\hline 1 & $8(4.7)$ & $3(1.8)$ \\
\hline 2 & $8(4.7)$ & $12(7.1)$ \\
\hline 3 & $14(8.1)$ & $12(7.1)$ \\
\hline 4 & $37(21.5)$ & $43(25.1)$ \\
\hline 5 & $105(61.0)$ & $99(58.6)$ \\
\hline Tumor Purity (\%) & 70 (IQR: 30-90) & 70 (IQR: 30-80) \\
\hline
\end{tabular}

Abbreviations: IQR, interquartile range; PSA, prostate-specific antigen; PCa, prostate cancer; BPH, benign prostate hyperplasia.

DHL5 was subclonal (Figure 4). Based on the imputation of cancer cell population, we could dissect the cancer progression and development among different loci.

\section{Discussion}

$\mathrm{PCa}$ is one of the highly heterogeneous tumors, which has been investigated by several studies, focusing on tumor evolution. ${ }^{17,18}$ Generally, tumor heterogeneity can be divided into two types: spatial and temporal heterogeneity. These two features are particularly obvious in PCa. As known to all, $\mathrm{PCa}$ is frequently multifocal; it has been reported that nearly $90 \%$ of radical prostatectomy specimens have multifocal disease, and this is related with higher grade, stage and recurrence rate than unifocal $\mathrm{PCa}{ }^{19,20}$ Ruijter et $\mathrm{al}^{21}$ investigated radical prostatectomy specimens with clinical stage $\mathrm{T} 2$ and found that $72 \%$ of the specimens had more than two foci and $84 \%$ had multiple grades. In another study, $87 \%$ of all specimens were presented with more than two adenocarcinoma foci. Further, they analyzed the correlation of Gleason score between the largest foci 


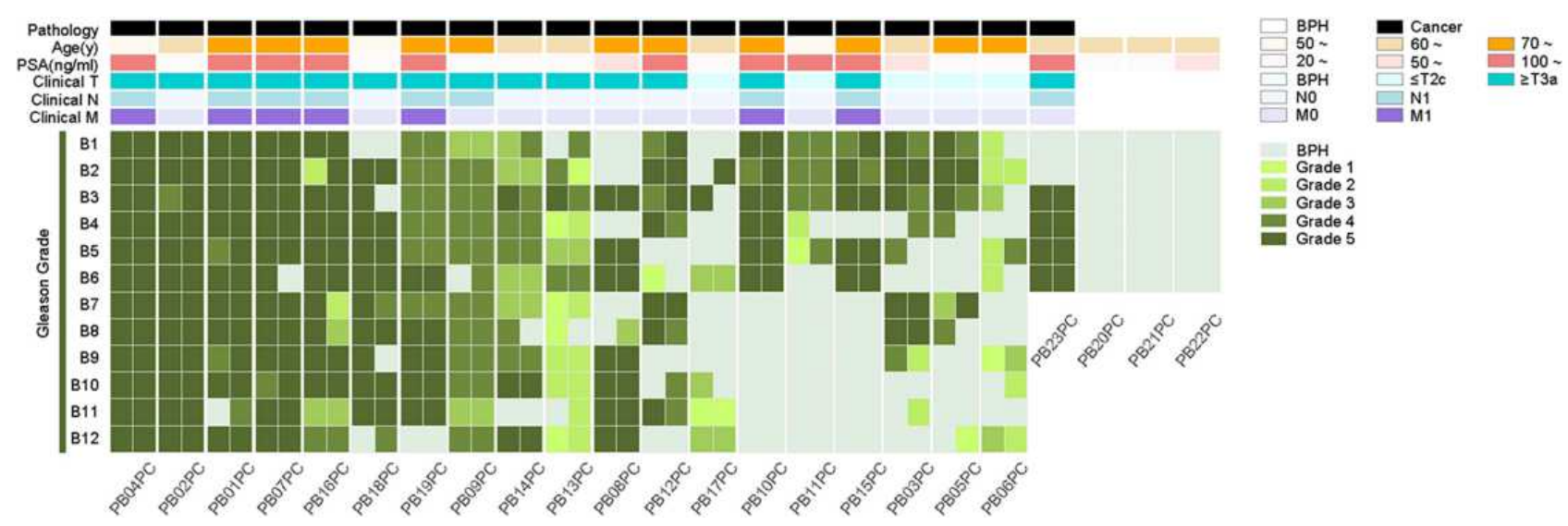

Figure I The clinical and pathological characters of each patient. The green bar below each patient shows the consistency of two biopsies.

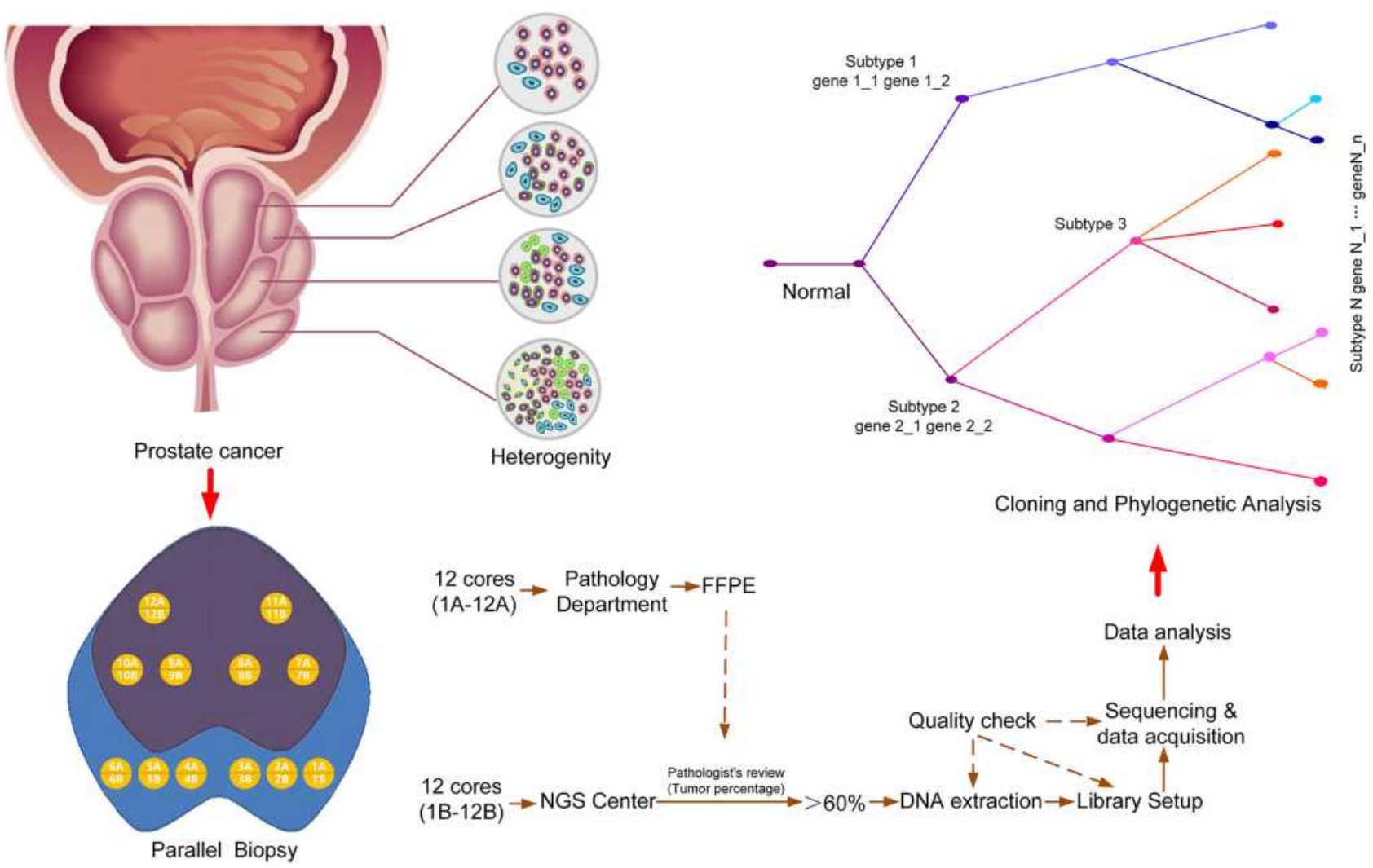

Figure 2 The overall conception of application of parallel biopsy in precise treatment of prostate cancer.

and overall score, discovering that only two-thirds of the specimens were correlated, and revealing histologic heterogeneity of multifocal prostate malignancies. ${ }^{22}$ Many studies have already analyzed the spatial genomic heterogeneity by NGS. Paul C Boutros et al ${ }^{18}$ investigated the spatial genomic heterogeneity within localized, multifocal $\mathrm{PCa}$, finding significant intraprostatic heterogeneity at the level of single- nucleotide variants, copy number aberrations and genomic rearrangement. In a recent study, researchers collected a large-scale set of frozen tissue samples that were taken from two-three distinct tumor foci and one normal area from 41 patients who received radical prostatectomy. A very high percentage of interfocal heterogeneity was found by whole-exome sequencing, revealing that there 


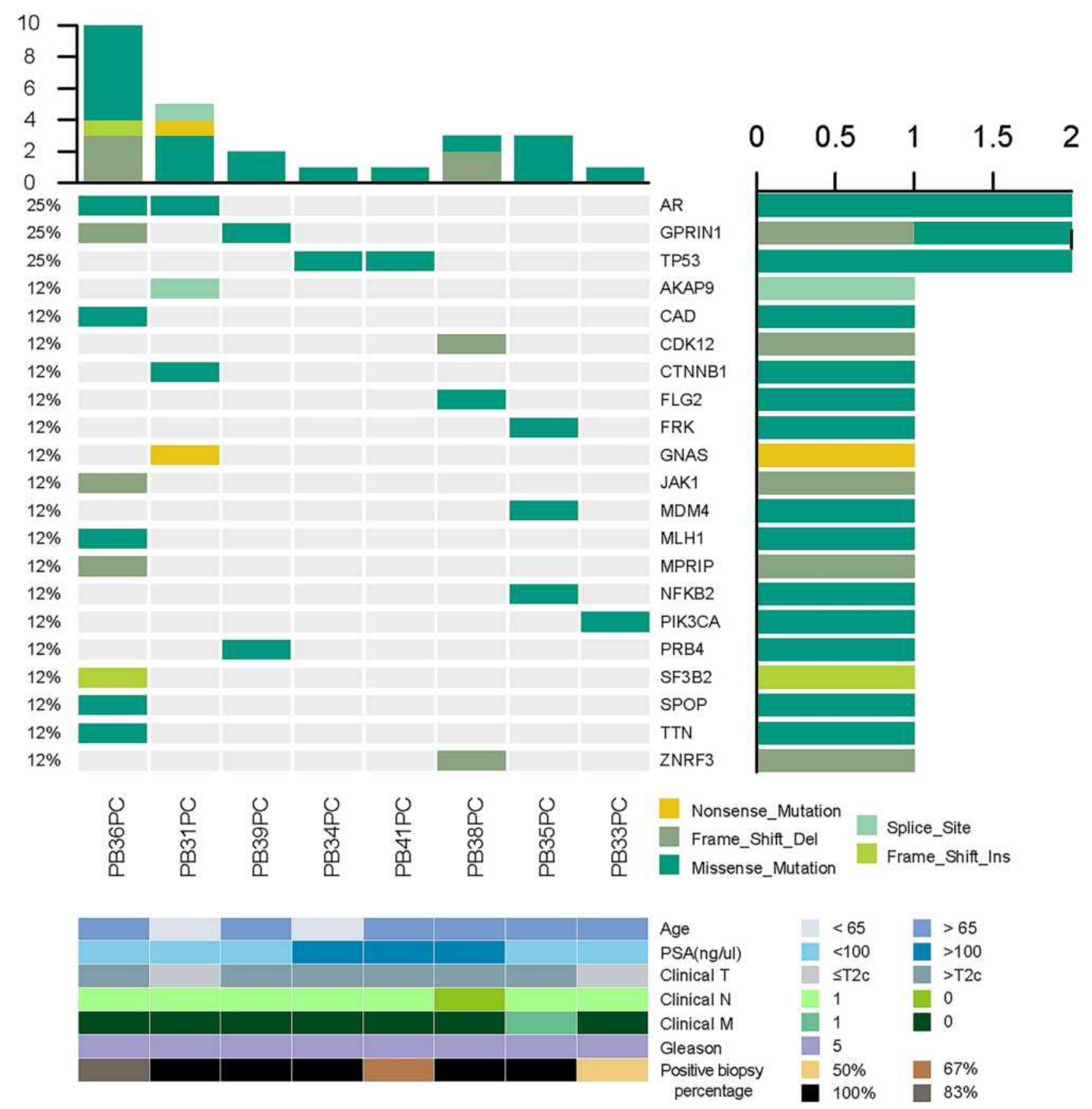

Figure 3 Molecular landscape of 8 patients sequenced by targeted gene panels. The patient named PB4IPC was sequenced by Pro24I-Panel, and others by 52I-Panel.

were no point mutations in common and DNA copy number variations were rarely shared across cancer foci.

Temporal heterogeneity is also a significant feature of PCa. Androgen deprivation therapy (ADT) is proved to be standard, providing initial benefit, but the majority of patients will progress to castration-resistant prostate cancer (CRPC) after 2-3 years. ${ }^{23}$ Although numerous studies have introduced and verified the effectiveness of novel agents such as abiraterone acetate (AA), an inhibitor of androgen synthesis, and enzalutamide, a potent antiandrogen, the state of disease remains incurable and is associated with significant morbidity and mortality. ${ }^{24-26}$ In the process from hormone-naïve to hormone-resistant, the general histological and molecular alteration indicates the temporal heterogeneity in PCa. Neuroendocrine differentiation is considered as one of the mechanisms of CRPC. It is reported that about $17-25 \%$ of patients with metastatic CRPC present with treatment-induced neuroendocrine 

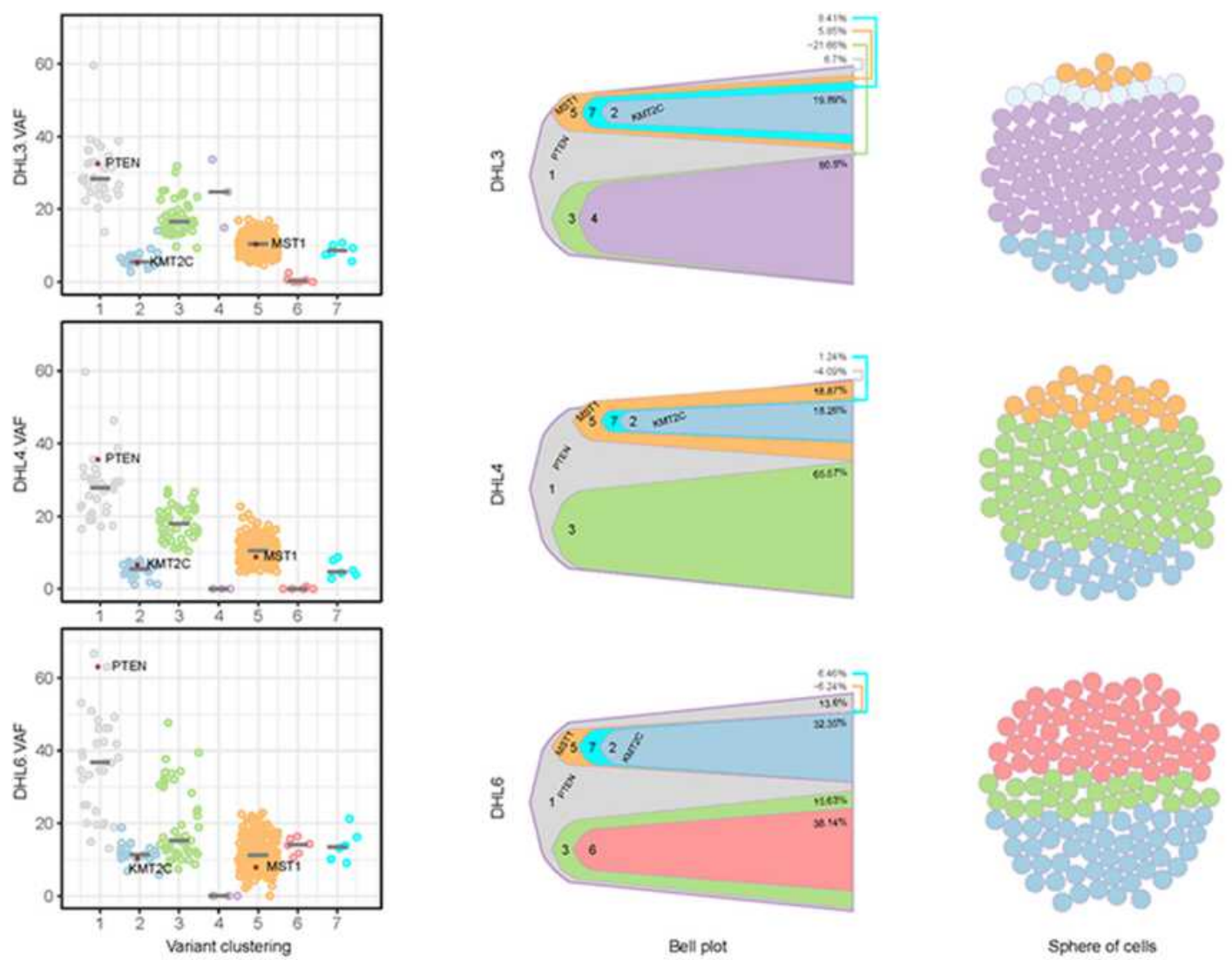

Bell plot

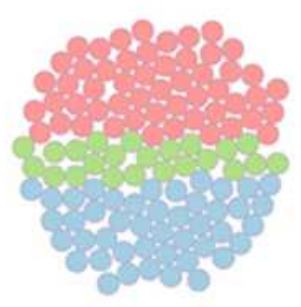

Sphere of cells

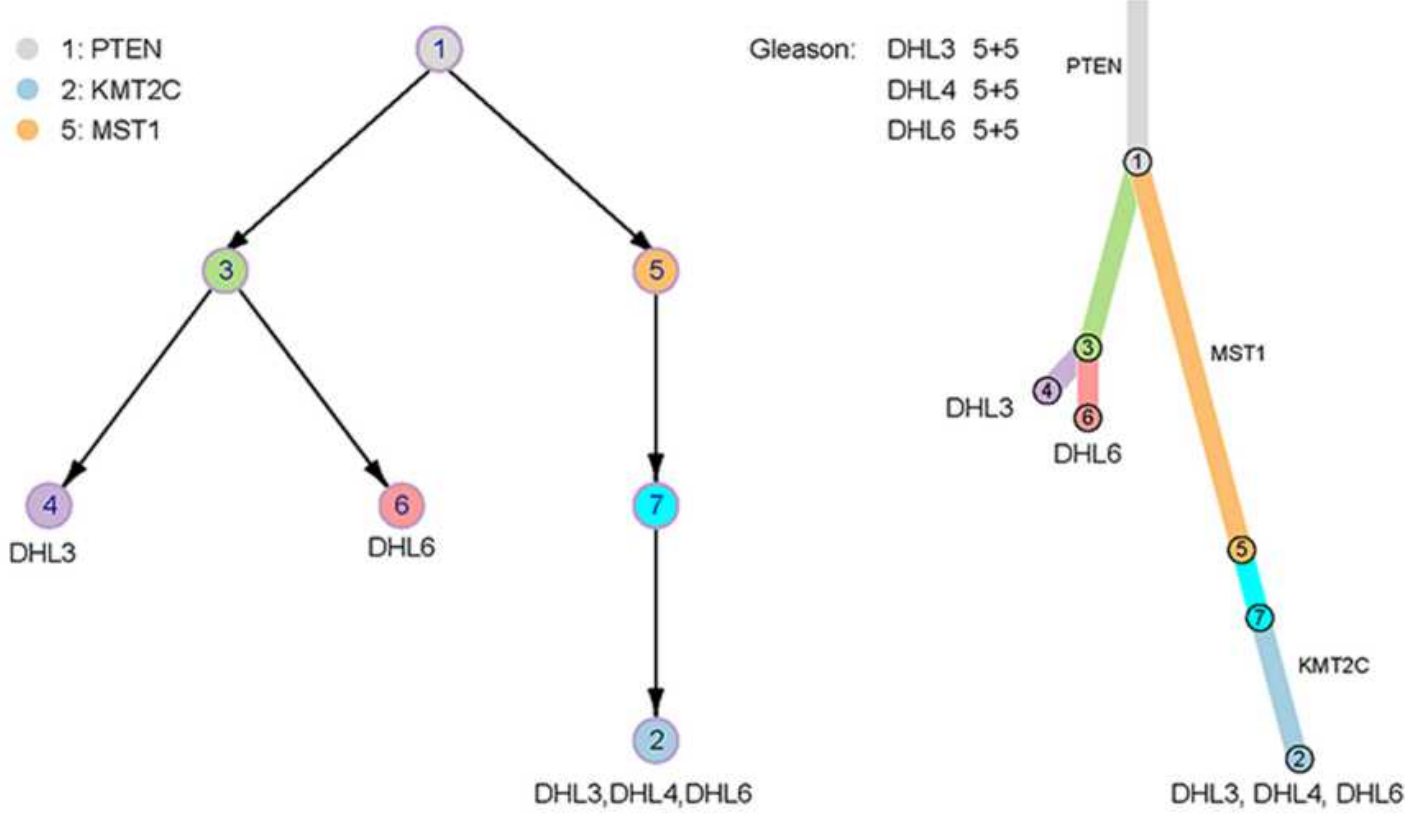

Node-based tree

Branch-based tree

Figure 4 Tumor clonal evolutionary tree. Bell plot and sphere of cells present the dynamics and proportion of clonal subpopulations, respectively. The node-based and branch-based trees describe the clonal relationships and seeding patterns. 
differentiation of $\mathrm{PCa}$, associated with poor overall survival. $^{27,28}$ Morphologically, it features small cell carcinoma and typically low or absent AR expression. Clinically, it presents with low PSA level, extensive visceral metastasis, osteolytic lesions and shortened response duration to ADT. Genetically, several studies have revealed MYCN and AURKA overexpression/gene amplification by using RNA sequencing. ${ }^{29}$ Further, Rb protein loss, TP53 accumulation, p16 overexpression and cyclin D1 loss were observed in more than half of the cases, indicating all these genetic alterations are related to the development of neuroendocrine differentiation in $\mathrm{PCa} .{ }^{30-32} \mathrm{~A}$ recent study analyzed whole-genome sequencing data of metastatic lesions from patients with CRPC, focusing on the clonal evolution pattern of neuroendocrine differentiation. The study performed sequential biopsies in the same patient, discovering a substantial genomic overlap between CRPC and neuroendocrine differentiation of $\mathrm{PCa}$. The results indicated that neuroendocrine differentiation arises from CRPC with obvious temporal heterogeneity.

Therefore, it is critical and urgent to invent an approach that can collect tumor tissue, covering the dimensions of time and space. Previously, a modified biopsy system was invented by milling two parallel rifts separated by a 100 $\mu \mathrm{m}$ thin sheet into the needle shaft based on BARD MaxCore System (MC1416). The pathology of double cores showed congruent results. ${ }^{33}$ However, the modified system is not available on the market, resulting in the difficulties of double tumor tissue sampling. We firstly propose the concept of parallel biopsy of the prostate, collecting the tissues from the same site by two biopsies. The results showed that tissues of parallel biopsy shared high consistency and could help constructing a clonal tree for further investigation of castration resistance. Otherwise, as prostate biopsy is performed under local anesthesia, one more puncture in a site does not add to the patient's pain and no one had any adverse events after punctures. We believe that the application of parallel biopsy can provide genotyping of the biopsy-based specimens collected from different foci or distinct time points from the same patient, deeply mining the biological mechanism of prostate cancer heterogeneity for further precision medicine.

However, there are still some limitations in this study. In the case of poor anesthesia, more needles may cause more pain. However, with the help of MRI before the procedure, it is now possible to predict pain levels and high-risk patients for pain. ${ }^{34}$ Otherwise, though prostate biopsy is the preferred method for diagnosing prostate cancer, the area we can sample on biopsy is very limited, and Gleason upgrade rates in radical prostatectomy are quite high. Therefore, applying fusion prostate biopsy to patients who will undergo targeted therapy or genetic studies may give more accurate results. ${ }^{20}$ However, due to the limitation of technique application, fusion biopsy by MRI-ultrasound is highly restricted in many countries. Thus, we believe that the parallel biopsy can be greatly applied where fusion biopsy is not popular enough, when precision medicine is needed.

\section{Abbreviations}

$\mathrm{PCa}$, prostate cancer; NGS, next-generation sequencing; $\mathrm{FF}$, fresh frozen; FFPE, formalin-fixed paraffin-embedded; WES, whole-exome sequencing; $\mathrm{BPH}$, benign prostatic hyperplasia; CRPC, castration-resistant prostate cancer; AA, abiraterone acetate; AURKA, aurora kinase A.

\section{Research Involving Human Participants}

This study protocol was approved by the Ethics Committee of Changhai Hospital and was in accordance with national research committee and with the 1964 Helsinki declaration and its later amendments or comparable ethical standards.

\section{Informed Consent}

Informed consent was obtained from all individual participants included in the study.

\section{Acknowledgments}

The authors thank all the patients who agreed to participate in this study.

\section{Funding}

This study is supported by National Natural Science Foundation of China (81602467, Jing Li); Sub-project under "Zhangiiang National Innovation Demonstration Zone" Initiative Development Fund (2017JZ17, Xu Gao); Shanghai Sailing Program (19YF1447000, Min Qu).

\section{Disclosure}

The authors report no conflicts of interest in this work.

\section{References}

1. Siegel RL, Miller KD, Jemal A. Cancer statistics, 2018. CA Cancer J Clin. 2018;68:7-30. doi:10.3322/caac.21442 
2. Andreoiu M, Cheng L. Multifocal prostate cancer: biologic, prognostic, and therapeutic implications. Hum Pathol. 2010;41:781-793. doi:10.1016/j.humpath.2010.02.011

3. Barbieri CE, Baca SC, Lawrence MS, et al. Exome sequencing identifies recurrent SPOP, FOXA1 and MED12 mutations in prostate cancer. Nat Genet. 2012;44:685-689. doi:10.1038/ng.2279

4. Fraser M, Sabelnykova VY, Yamaguchi TN, et al. Genomic hallmarks of localized, non-indolent prostate cancer. Nature. 2017;541:359-364. doi:10.1038/nature20788

5. Wedge DC, Gundem G, Mitchell T, et al. Sequencing of prostate cancers identifies new cancer genes, routes of progression and drug targets. Nat Genet. 2018;50:682-692. doi:10.1038/s41588-018-0086$\mathrm{z}$

6. Cornford P, van den Bergh RCN, Briers E, et al. EAU-EANMESTRO-ESUR-SIOG guidelines on prostate cancer. Part II-2020 update: treatment of relapsing and metastatic prostate cancer. Eur Urol. 2021;79:263-282. doi:10.1016/j.eururo.2020.09.046

7. Palermo G, Bassi P, Racioppi M, et al. Circulating tumor cells as prognostic biological marker in different stages prostate cancer and the effect of different therapeutic approaches on their expression. Minerva urologica e nefrologica. 2020;72:214-222. doi:10.23736/ S0393-2249.19.03377-0

8. Jiang Z, Zhao Y, Tian Y. Comparison of diagnostic efficacy by two urine PCA3 scores in prostate cancer patients undergoing repeat biopsies. Minerva urologica e nefrologica. 2019;71:373-380. doi:10.23736/S0393-2249.18.03093-X

9. Lovf M, Zhao S, Axcrona U, et al. Multifocal primary prostate cancer exhibits high degree of genomic heterogeneity. Eur Urol. 2019;75 (3):498-505. doi:10.1016/j.eururo.2018.08.009

10. Loeb S, Vellekoop A, Ahmed HU, et al. Systematic review of complications of prostate biopsy. Eur Urol. 2013;64:876-892. doi:10.1016/j.eururo.2013.05.049

11. De Paoli-iseppi R, Johansson PA, Menzies AM, et al. Comparison of whole-exome sequencing of matched fresh and formalin fixed paraffin embedded melanoma tumours: implications for clinical decision making. Pathology. 2016;48:261-266. doi:10.1016/j. pathol.2016.01.001

12. Hognas G, Kivinummi K, Kallio HML, et al. Feasibility of prostate PAXgene fixation for molecular research and diagnostic surgical pathology: comparison of matched fresh frozen, FFPE, and PFPE tissues. Am J Surg Pathol. 2018;42:103-115. doi:10.1097/ PAS.0000000000000961

13. Hubner N, Shariat S, Remzi M. Prostate biopsy: guidelines and evidence. Curr Opin Urol. 2018;28:354-359. doi:10.1097/ MOU.0000000000000510

14. Shen R, Seshan VE. FACETS: allele-specific copy number and clonal heterogeneity analysis tool for high-throughput DNA sequencing. Nucleic Acids Res. 2016;44:e131. doi:10.1093/nar/gkw520

15. Roth A, Khattra J, Yap D, et al. PyClone: statistical inference of clonal population structure in cancer. Nat Methods. 2014;11:396-398. doi: $10.1038 /$ nmeth.2883

16. Dang HX, White BS, Foltz SM, et al. ClonEvol: clonal ordering and visualization in cancer sequencing. Ann Oncol. 2017;28:3076-3082. doi:10.1093/annonc/mdx 517

17. Lindberg J, Klevebring D, Liu W, et al. Exome sequencing of prostate cancer supports the hypothesis of independent tumour origins. Eur Urol. 2013;63:347-353. doi:10.1016/j.eururo.2012.03.050

18. Boutros PC, Fraser M, Harding NJ, et al. Spatial genomic heterogeneity within localized, multifocal prostate cancer. Nat Genet. 2015;47:736-745. doi:10.1038/ng.3315
19. Djavan B, Susani M, Bursa B, et al. Predictability and significance of multifocal prostate cancer in the radical prostatectomy specimen. Tech Urol. 1999;5:139-142. doi:10.1016/S0022-5347(01)62084-6

20. Demirtas A, Sonmez G, Tombul ST, et al. Comparison of the upgrading rates of international society of urological pathology grades and tumor laterality in patients undergoing standard 12-core prostate biopsy versus fusion prostate biopsy for prostate cancer. Urol Int. 2019;103:256-261. doi:10.1159/000501528

21. Ruijter ET, van de Kaa CA, Schalken JA, et al. Histological grade heterogeneity in multifocal prostate cancer. Biological and clinical implications. J Pathol. 1996;180:295-299. doi:10.1002/(SICI)10969896(199611)180:3<295::AID-PATH663>3.0.CO;2-W

22. Arora R, Koch MO, Eble JN, et al. Heterogeneity of Gleason grade in multifocal adenocarcinoma of the prostate. Cancer. 2004;100:2362-2366. doi:10.1002/cncr.20243

23. Harris WP, Mostaghel EA, Nelson PS, et al. Androgen deprivation therapy: progress in understanding mechanisms of resistance and optimizing androgen depletion. Nat Clin Pract Urol. 2009;6:76-85. doi:10.1038/ncpuro1296

24. de Bono JS, Logothetis CJ, Molina A, et al. Abiraterone and increased survival in metastatic prostate cancer. $N$ Engl $\mathrm{J} \mathrm{Med}$. 2011;364:1995-2005. doi:10.1056/NEJMoa1014618

25. Scher HI, Fizazi K, Saad F, et al. Increased survival with enzalutamide in prostate cancer after chemotherapy. $N$ Engl J Med. 2012;367:1187-1197. doi:10.1056/NEJMoa1207506

26. Toren PJ, Gleave ME. Evolving landscape and novel treatments in metastatic castrate-resistant prostate cancer. Asian $J$ Androl. 2013;15:342-349. doi:10.1038/aja.2013.38

27. Aggarwal R, Huang J, Alumkal JJ, et al. Clinical and genomic characterization of treatment-emergent small-cell neuroendocrine prostate cancer: a multi-institutional prospective study. $J$ Clin Oncol. 2018;36:2492-2503. doi:10.1200/JCO.2017.77.6880

28. Aparicio A, Logothetis CJ, Maity SN. Understanding the lethal variant of prostate cancer: power of examining extremes: figure 1. Cancer Discov. 2011;1:466-468. doi:10.1158/2159-8290.CD-110259

29. Beltran H, Rickman DS, Park K, et al. Molecular characterization of neuroendocrine prostate cancer and identification of new drug targets. Cancer Discov. 2011;1:487-495. doi:10.1158/2159-8290.CD-110130

30. Aparicio AM, Shen L, Tapia EL, et al. Combined tumor suppressor defects characterize clinically defined aggressive variant prostate cancers. Clin Cancer Res. 2016;22:1520-1530. doi:10.1158/10780432.CCR-15-1259

31. Tan HL, Sood A, Rahimi HA, et al. Rb loss is characteristic of prostatic small cell neuroendocrine carcinoma. Clin Cancer Res. 2014;20:890-903. doi:10.1158/1078-0432.CCR-13-1982

32. Tsai H, Morais CL, Alshalalfa M, et al. Cyclin D1 loss distinguishes prostatic small-cell carcinoma from most prostatic adenocarcinomas. Clin Cancer Res. 2015;21:5619-5629. doi:10.1158/1078-0432.CCR15-0744

33. Kronig M, Nanko N, Drendel V, et al. Single punch, double biopsy. SpringerPlus. 2016;5:1456. doi:10.1186/s40064-016-3141-1

34. Sonmez G, Tombul ST, Demirtas T, et al. Risk factors associated with pain in fusion prostate biopsy. Prostate Int. 2020;8:185-189. doi:10.1016/j.prnil.2020.05.004 


\section{Publish your work in this journal}

The International Journal of General Medicine is an international, peer-reviewed open-access journal that focuses on general and internal medicine, pathogenesis, epidemiology, diagnosis, monitoring and treatment protocols. The journal is characterized by the rapid reporting of reviews, original research and clinical studies across all disease areas. The manuscript management system is completely online and includes a very quick and fair peer-review system, which is all easy to use. Visit http://www.dovepress.com/ testimonials.php to read real quotes from published authors.

Submit your manuscript here: https://www.dovepress.com/international-journal-of-general-medicine-journal 